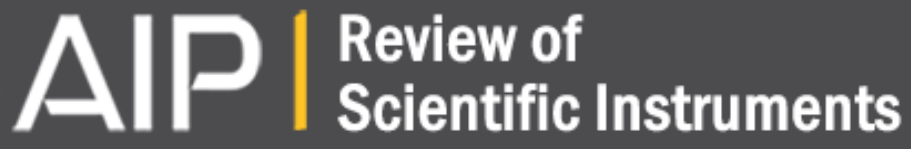

\section{A common-path heterodyne interferometer for surface profiling in microelectronic fabrication}

Eric J. Klein, W. Fred Ramirez, and John L. Hall

Citation: Review of Scientific Instruments 72, 2455 (2001); doi: 10.1063/1.1367353

View online: http://dx.doi.org/10.1063/1.1367353

View Table of Contents: http://scitation.aip.org/content/aip/journal/rsi/72/5?ver=pdfcov

Published by the AIP Publishing

\section{Articles you may be interested in}

Improved common-path fast-scanning heterodyne interferometer system as potential dense-plasma diagnostics Rev. Sci. Instrum. 75, 3417 (2004); 10.1063/1.1786639

Power spectral densities: A multiple technique study of different Si wafer surfaces

J. Vac. Sci. Technol. B 20, 31 (2002); 10.1116/1.1428267

Picosecond photoacoustics using common-path interferometry

Appl. Phys. Lett. 76, 514 (2000); 10.1063/1.125805

Error budget of step height and pitch measurement using a scanning tunneling microscope with a threedimensional interferometer

J. Vac. Sci. Technol. B 15, 1494 (1997); 10.1116/1.589481

A prototype imaging second harmonic interferometer

Rev. Sci. Instrum. 68, 709 (1997); 10.1063/1.1147681

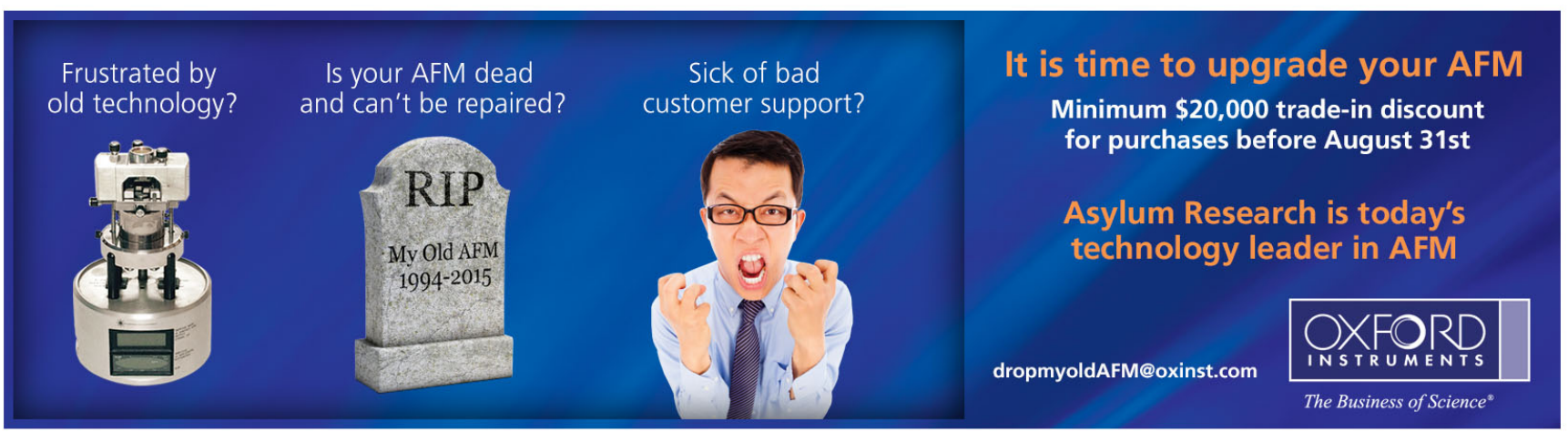




\title{
A common-path heterodyne interferometer for surface profiling in microelectronic fabrication
}

\author{
Eric J. Klein and W. Fred Ramirez ${ }^{\mathrm{a})}$ \\ Department of Chemical Engineering, University of Colorado, Campus Box 424, Boulder, \\ Colorado 80309-0424 \\ John L. Hall \\ JILA, University of Colorado and National Institute of Standards and Technology, Boulder, \\ Colorado 80309-0440
}

(Received 8 June 2000; accepted for publication 26 February 2001)

\begin{abstract}
We describe the design of a common-path heterodyne laser interferometer for the surface profiling of micron-sized photopatterned features during the microelectronic fabrication process. The common-path design of the interferometer's reference and measurement arms effectively removes any path length difference in the measurement which can be attributed to the movement of the target surface. It is shown that repeated surface profiling during the ion milling process allows the difference in etch rates between the photoresist layer and the exposed portions of the underlying substrate layer to be monitored online. A prototype apparatus has been assembled and results demonstrating the usefulness of the device are reported. The surface profiles of both a photopatterned nickel-iron trench and an unmasked aluminum trench are measured and compared to those obtained using a stylus-based scanning profiler and an atomic force microscope. (C) 2001 American Institute of Physics. [DOI: 10.1063/1.1367353]
\end{abstract}

\section{INTRODUCTION}

The physical etching of patterned wafers through ion bombardment is a crucial step in the fabrication of many microelectronic devices. Patterns are transferred to the wafer surface using photolithography techniques such that the resulting photoresist layer physically protects desired portions of the wafer surface from the ion beam during milling. State of the art techniques for monitoring ion milling etch depth in situ are inadequate, making the online identification of process faults a difficult if not impossible task. Most ion milling processes therefore produce an unacceptably high percentage of nonconforming parts which frequently cause the failure of the fabricated device. To meet the current demands of the microelectronic industry and the continued trends towards smaller, more intricate surface features, a device capable of monitoring in situ etch depth in homogeneous material layers with a resolution on the order of $1 \mathrm{~nm}$ is needed.

Currently, online etch depth monitoring is largely limited to end point detectors, most of which are based on secondary ion mass spectrometry (SIMS) techniques. ${ }^{1-5}$ These SIMS-based end point detectors, which monitor the composition of sputtered atoms, are useful for determining when boundaries between material layers have been reached during the milling of multilayer surfaces. However, SIMS-based techniques are inherently incapable of determining absolute etch depth in homogeneous material layers.

In addition, several researchers have reported on the use of laser reflectometry for the in situ monitoring of etch depth. ${ }^{6-10}$ In laser reflectometry the reflected light from the top and underlying material layers of multilayer wafer sur-

\footnotetext{
a) Author to whom correspondence should be addressed; electronic mail: fred.ramirez@colorado.edu
}

faces interfere. The interference causes a sinusoidal pattern in the reflectance signal, the period of which corresponds to a thickness change in the top layer equal to one half of a wavelength in the material ${ }^{11}$

$$
\Delta d=\frac{\lambda}{2 n},
$$

where $\Delta d$ is the change in thickness, $\lambda$ is the wavelength of the light employed, and $n$ is the refractive index of the exposed material layer. When visible light is employed, this technique exhibits a resolution on the order of $100 \mathrm{~nm}$ which is not adequate for monitoring most ion milling applications. Furthermore, the resolution declines sharply when the wafer materials are highly absorbent at the wavelength of the laser, which at visible wavelengths is the case for many materials common to the microelectronics industry.

It is possible, however, to make use of the fact that in ion milling processes, in contrast to wet etching processes where the photoresist is chemically resistant to the etching medium, both the photoresist layer and the exposed portions of the wafer surface are etched. The etch rates of the two materials are based on the physical properties of each material layer and are in general significantly different. We have shown elsewhere that knowledge of the difference in these etch rates during the milling process provides information that is adequate for making optimal state estimations in the presence of system disturbances. ${ }^{12}$ A fundamental model of the ion milling process, which requires precise knowledge of the initial wafer surface contours, has also been described elsewhere. ${ }^{13}$ Optimal estimations of the process states used in conjunction with the process model can be used to accurately predict in situ etch depth and thereby precisely regulate final etch depth in the ion milling process. 


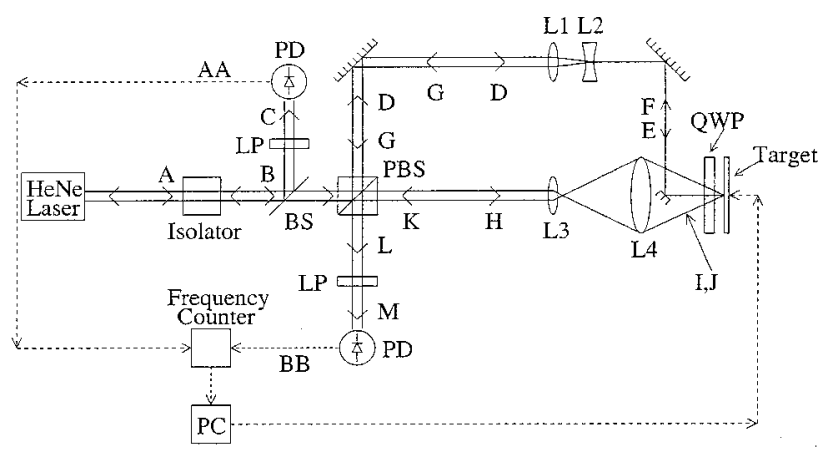

FIG. 1. Schematic diagram of the common-path heterodyne interferometer: (PD) photodetector, (QWP) quarter wave plate, (LP) linear polarizer, (BS) beam splitter, (PBS) polarizing beam splitter, (PC) personal computer.

We propose and demonstrate the use of a common-path heterodyne laser interferometer to provide the initial surface profiles of photopatterned wafer features required by the process model as well as in situ measurements of trench height required by the optimal state estimation system. Several researchers have described the use of heterodyne scanning interferometers for measuring absolute distance and surface profiles with resolutions on the order of $1 \mathrm{~nm} .{ }^{14-17}$ The usefulness of common-path beam design for removing path length changes due to vibration of the target surface has also been discussed. ${ }^{18}$ The common-path design is crucial to the application at hand since wafers are typically rotated in the azimuth plane during milling which results in vibration of the wafer surface. Repeated surface profiling during the milling process will provide for the in situ monitoring of the difference in etch rates between the photoresist and the exposed portion of the wafer surface.

The remainder of this article reviews the applicable theory and details the design of a prototype apparatus for monitoring etch rates in the ion milling process. Testing of the apparatus, which consisted of profiling both photopatterned and unmasked surface features of a wafer mounted on a translational stage, has been completed and the results are reported herein. It is demonstrated that the device is able to repeatedly provide accurate surface profiles with a resolution on the order of $1 \mathrm{~nm}$ while minimizing noise due to sample vibration.

\section{THEORY}

\section{A. Interferometer design}

A detailed schematic of the common-path heterodyne interferometer is depicted in Fig. 1. The device utilizes a Hewlett-Packard (Santa Clara, CA) model HP-5500 heterodyne $\mathrm{HeNe}$ laser head, which provides two coaxial, 632.8 $\mathrm{nm}$ wavelength beams with radii of $w_{0}=2 \mathrm{~mm}$ (beam A). The coaxial beams are orthogonally linearly polarized and exhibit a nominal Zeeman split optical frequency difference of $1.8 \mathrm{MHz}$. The coaxial beams pass through a Faraday isolator, which consists of a half-wave plate and a Faraday rotation device, to minimize feedback to the laser.

Beam $B$ then travels to a beam splitter. The reflected portion of beam B passes through a linear polarizer oriented at $45^{\circ}$ such that the orthogonally polarized coaxial beams are projected to a common polarization state, resulting in beam C. Beam C is sent to a photodetector.

We can describe the electric fields of the two coherent coaxial beams as follows:

$$
\begin{aligned}
& E_{1}=A_{1} \sin \left(\omega_{1} t+\phi_{1}\right), \\
& E_{2}=A_{2} \cos \left(\omega_{2} t+\phi_{2}\right),
\end{aligned}
$$

where $A_{1}$ and $A_{2}$ are the amplitudes, $\omega_{1}$ and $\omega_{2}$ the angular frequencies, and $\phi_{1}$ and $\phi_{2}$ the phases of the two beams, respectively. In heterodyne detection the intensity at the photodetector varies as $\eta\left|E_{1}+E_{2}\right|^{2}$, where $\eta$ is the photodetector responsivity. The output current thus contains a heterodyne cross term $\eta E_{1} E_{2}^{*}$ in addition to the two dc currents $\eta\left|E_{1}\right|^{2}$ and $\eta\left|E_{2}\right|^{2}$. That is,

$$
I_{1}=\eta\left|E_{1}+E_{2}\right|^{2}=\eta\left(\left|E_{1}\right|^{2}+\left|E_{2}\right|^{2}+2 E_{1} E_{2}^{*}\right) .
$$

The ac signal-bearing portion of the intensity is therefore

$$
\begin{aligned}
I_{1, \mathrm{ac}} & \propto E_{1} E_{2}^{*} \\
& \propto \sin \left(\omega_{1} t+\phi_{1}\right) \cos \left(\omega_{2} t+\phi_{2}\right) \\
& \propto \frac{1}{2} \sin \left(\omega_{1} t-\omega_{2} t+\phi_{1}-\phi_{2}\right)+\frac{1}{2} \sin \left(\omega_{1} t+\omega_{2} t+\phi_{1}+\phi_{2}\right) .
\end{aligned}
$$

The phase terms of Eq. (7) cancel since the path lengths of the two concentric beams are equal. Furthermore, the photodetector is unable to detect the high frequency of the second term of Eq. (7), so that the output from the photodetector (signal AA in Fig. 1) can be expressed as

$$
\mathrm{AA} \propto \sin \left[\left(\omega_{1}-\omega_{2}\right) t\right] .
$$

Signal AA, which exhibits the heterodyne split frequency of $1.8 \mathrm{MHz}$, is sent through a 1.6-3.0 MHz bandpass filter and to a Hewlett Packard (Santa Clara, CA) model HP-53132A frequency counter as the reference for phase detection.

The transmitted portion of beam B is sent to a polarizing beam splitter cube, which splits the coaxial heterodyne beam $\mathrm{B}$ into the reference and measurement arms of the modified Michelson interferometer, beams D and H, respectively. Reference beam $\mathrm{D}$, which is linearly $s$ polarized, passes through a converging lens $(L 1$, focal length $=30 \mathrm{~cm}$, diameter $=2.5$ $\mathrm{cm}$ ) and a diverging lens ( $L 2$, focal length $=-10 \mathrm{~cm}$, diameter $=2.5 \mathrm{~cm}$ ) resulting in beam $\mathrm{E}$, which is reduced to a beam radius of $w_{0}=200 \mu \mathrm{m}$. Three mirrors facilitate the beam steering of beam $\mathrm{D} / \mathrm{E}$ to the target surface. Beam $\mathrm{E}$ passes through a quarter-wave plate, reflects off of the target surface, and passes back through the quarter-wave plate resulting in beam $\mathrm{F}$ which is linearly $p$ polarized. Beam $\mathrm{F}$ travels back through the telescope assembly resulting in beam $\mathrm{G}$, which is of the original beam radius $w_{0}=2 \mathrm{~mm}$ and linearly $p$ polarized such that it is transmitted through the polarizing beam splitter cube.

The measurement beam $\mathrm{H}$, which is linearly $p$ polarized, passes through a converging lens ( $L 3$, focal length $=4 \mathrm{~cm}$, diameter $=2.5 \mathrm{~cm}$ ) where it is focused to a spot size of $w_{0}$ $=4 \mu \mathrm{m}$. A second lens assembly $(L 4)$ performs a 1:1 imaging of the $4 \mu \mathrm{m}$ spot, resulting in beam I. This second lens assembly ( $L 4)$ is comprised of two achromat lenses (focal length $=40 \mathrm{~cm}$, diameter $=7.5 \mathrm{~cm}$ ) in order to reduce the 


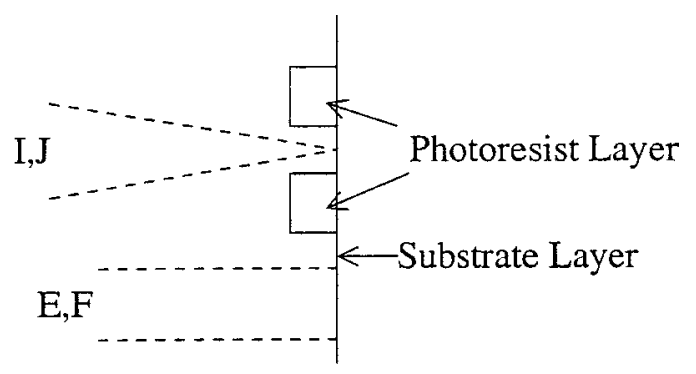

FIG. 2. Enlarged depiction of the interferometer beam design, showing measurement beam $\mathrm{I} / \mathrm{J}$ in close proximity to reference beam $\mathrm{E} / \mathrm{F}$.

effects of spherical aberration at the focal point on the wafer surface. The large focal length is required in practice to enable the beam to be sent into the ion milling vacuum chamber via an appropriate view port. Beam I travels through the quarter-wave plate, reflects off of the target surface, and travels back through the quarter-wave plate resulting in beam $\mathrm{J}$, which is linearly $s$ polarized. Beam $\mathrm{J}$ travels back through the imaging system resulting in beam $\mathrm{K}$, which is of the original beam diameter $w_{0}=2 \mathrm{~mm}$ and is linearly $s$ polarized such that it is reflected by the polarizing beam splitter cube.

Surface profiles are obtained by mounting the wafer on a translational stage which is controlled by a personal computer to allow the raster scanning of measurement beam I and reference beam $\mathrm{E}$ across the wafer surface. The design is such that the measurement beam I and reference beam $\mathrm{E}$ are in close proximity to each other, spaced approximately $<2$ $\mathrm{mm}$ apart (Fig. 2). The close proximity of the interferometer arms facilitates the removal of path length difference due to target vibration from the measurement. During the scan, reference beam $E$ remains focused on the relatively smooth substrate layer and therefore does not see the photopatterned surface features.

Beams $\mathrm{G}$ and $\mathrm{K}$ are coaxial and orthogonally linearly polarized upon leaving the polarizing beam splitter cube. This orthogonal polarization present in beam $\mathrm{L}$ is mixed using a linear polarizer oriented at $45^{\circ}$. The mixed beam $\mathrm{M}$ is sent to a second photodetector. The electric fields of the coaxial beams that comprise beam $\mathrm{M}$ can be described as follows:

$$
\begin{aligned}
& E_{\text {ref }}=A_{\text {ref }} \sin \left(\omega_{\text {ref }} t+\phi_{0}+\phi_{\text {ref }}\right), \\
& E_{\text {meas }}=A_{\text {meas }} \cos \left(\omega_{\text {meas }} t+\phi_{0}+\phi_{\text {meas }}\right),
\end{aligned}
$$

where $\phi_{0}$ is the phase associated with target vibration. Here $\phi_{\text {ref }}$ is the return phase of the reference beam, averaged over the surface roughness explored within the beam's diameter. Similarly $\phi_{\text {meas }}$ is the averaged phase of the returned measurement beam, including the phase changes due to the length changes we intend to associate with the contours of the surface. The ac portion of the intensity at the second photodetector can be written as

$$
\begin{aligned}
I_{2, \mathrm{ac}} & \propto E_{\text {ref }} E_{\text {meas }} \\
\propto & \sin \left(\omega_{\text {ref }} t+\phi_{0}+\phi_{\text {ref }}\right) \cos \left(\omega_{\text {meas }} t+\phi_{0}+\phi_{\text {meas }}\right) \\
\propto & \frac{1}{2} \sin \left(\omega_{\text {ref }} t+\phi_{0}+\phi_{\text {ref }}-\left(\omega_{\text {meas }} t+\phi_{0}+\phi_{\text {meas }}\right)\right) \\
& +\frac{1}{2} \sin \left(\omega_{\text {ref }} t+\omega_{\text {meas }} t+2 \phi_{0}+\phi_{\text {ref }}+\phi_{\text {meas }}\right) .
\end{aligned}
$$

Again, the photodetector is unable to detect the high frequency of the second term of Eq. (13), so that the output from the photodetector, signal BB in Fig. 1, can be given as

$$
\mathrm{BB} \propto \sin \left[\left(\omega_{\text {ref }}-\omega_{\text {meas }}\right) t+\left(\phi_{\text {ref }}-\phi_{\text {meas }}\right)\right] .
$$

Note that the phase due to target vibration $\phi_{0}$ is not present in the detector output. The validity of the ordering of terms in Eq. (14) has been verified by heterodyning the measurement and reference beams with an external $\mathrm{He}-\mathrm{Ne}$ laser source. That is, it has been verified that $\omega_{\text {ref }}>\omega_{\text {meas }}$.

Signal BB is sent through a $1.6-3.0 \mathrm{MHz}$ band pass filter and a $20 \mathrm{~dB}$ amplifier to the frequency counter where the phase of signal AA with respect to signal BB is measured. This measured phase $\phi_{m}$ is sent to a personal computer for analysis.

\section{B. Phase analysis}

The measured phase $\phi_{m}$ is indicative of the distance traveled by the measurement beam during the scanning of the wafer surface with respect to the surface, averaged over its roughness, within the diameter of the reference beam. The surface profile is reconstructed by monitoring this phase as the laser scans across the wafer surface.

The phases of signal BB at two discrete surface positions $x_{1}$ and $x_{2}$ are as follows:

$$
\begin{aligned}
& \phi_{\mathrm{BB}, x_{1}}=\phi_{\text {ref }, x_{1}}-\phi_{\text {meas }, x_{1}}, \\
& \phi_{\mathrm{BB}, x_{2}}=\phi_{\text {ref }, x_{2}}-\phi_{\text {meas }, x_{2}} .
\end{aligned}
$$

Assuming that the surface roughness of the relatively smooth substrate layer is sufficiently small and random such that the path length of the reference arm remains relatively unchanged during the raster scan, we can write

$$
\Delta \phi_{\mathrm{BB}}=\phi_{\mathrm{BB}, x_{2}}-\phi_{\mathrm{BB}, x_{1}}=\phi_{\text {meas }, x_{1}}-\phi_{\text {meas }, x_{2}} .
$$

The measured phase $\phi_{m}$ is the phase of signal AA with respect to signal $\mathrm{BB}$ so that the change in measured phase can be expressed as

$$
\Delta \phi_{m}=-\Delta \phi_{\mathrm{BB}}=\phi_{\text {meas }, x_{2}}-\phi_{\text {meas }, x_{1}} .
$$

Note that the change in measured phase is independent of both the average surface roughness and any path length difference associated with vibration of the wafer surface.

In general, the magnitude of the surface height difference between points $x_{1}$ and $x_{2}$ is deduced as follows. The one-way optical path lengths of the measurement arm at points $x_{1}$ and $x_{2}$ are written as ${ }^{17}$

$$
\begin{aligned}
& L_{x_{1}}=\left(N_{x_{1}}+\epsilon_{x_{1}}\right) \lambda / 2, \\
& L_{x_{2}}=\left(N_{x_{2}}+\epsilon_{x_{2}}\right) \lambda / 2, \\
& \epsilon_{x_{1}}=\frac{-\phi_{\text {meas }, x_{1}}}{2 \pi}, \\
& \epsilon_{x_{2}}=\frac{-\phi_{\text {meas }, x_{2}}}{2 \pi},
\end{aligned}
$$


where $N$ is the integral fringe number and $\epsilon$ is the fractional fringe number. The right hand sides of Eqs. (19) and (20) are divided by 2 since the beams of the interferometer travel both to and from the wafer surface.

In the present implementation, the fractional fringe number cannot be determined explicitly from the phase measurement due to the lack of knowledge of $\phi_{\text {ref }}$. However, the change in the fractional fringe number between points $x_{1}$ and $x_{2}$ is determined directly from the change in the phase measurement

$$
\begin{aligned}
\Delta \epsilon & =\frac{\epsilon_{x_{2}}-\epsilon_{x_{1}}}{2 \pi} \\
& =\frac{\phi_{\text {meas }, x_{1}}-\phi_{\text {meas }, x_{2}}}{2 \pi} \\
& =\frac{-\Delta \phi_{m}}{2 \pi}
\end{aligned}
$$

such that the change in path length can be expressed as follows:

$$
\begin{aligned}
\Delta L & =L_{x_{2}}-L_{x_{1}} \\
& =(\Delta N+\Delta \epsilon) \lambda / 2 \\
& =\left(\Delta N-\Delta \phi_{m} / 2 \pi\right) \lambda / 2,
\end{aligned}
$$

where

$$
\Delta N=N_{x_{2}}-N_{x_{1}} .
$$

While the change in the fractional fringe number, which is related to the change in the measured phase angle, is measured precisely, determination of the change in the integral fringe number $\Delta N$ is somewhat more difficult. This can be accomplished through basic fringe counting techniques in cases where the surface profile does not exhibit discontinuities, or through a priori knowledge of the feature height within $\pm \lambda / 4 .{ }^{17}$ Optionally, the integer fringe number $N$ can be determined by employing multiwavelength light. ${ }^{14}$ In the case of the experiments performed in this study (Sec. III), and when using the heterodyne device to provide initial surface profiles required by the process model, ${ }^{13}$ the height of the surface feature is known within $\pm \lambda / 4$ a priori so that determination of the change in integer fringe number is not a problem.

Furthermore, the difference in ion milling etch rates between the photoresist and wafer surface layers is sufficiently small (on the order of $0.5 \mathrm{~nm} / \mathrm{s}$ ) that the integral fringe number difference between the top and bottom surfaces of a photopatterned trench will not change over the course of a typical run. This makes the determination of the integral fringe number for in situ etch rate monitoring applications a nonissue. This can be seen by letting $h_{1}$ and $h_{2}$ be the trench height at times $t_{1}$ and $t_{2}$, respectively, and expressing the differential etch rate as

$$
\begin{aligned}
\Delta q & =\left(h_{2}-h_{1}\right) /\left(t_{2}-t_{1}\right) \\
& =\left(\Delta L_{2}-\Delta L_{1}\right) /\left(t_{2}-t_{1}\right) \\
& =\left(\Delta \phi_{\text {meas }, 1}-\Delta \phi_{\text {meas }, 2}\right) \lambda / 4 \pi\left(t_{2}-t_{1}\right),
\end{aligned}
$$

which is independent of the change in integral fringe number and is determined directly from the available phase measurements.

Note that in cases where the measurement spot is split between the top and bottom surfaces of the trench that the measured phase angle lies between the phase angle associated with the top surface $\left(\phi_{\text {meas, } 1}\right)$ and that of the bottom surface $\left(\phi_{\text {meas }, 2}\right)$. In this case the power is divided according to area and the more general expressions for the electric fields of the measurement and reference beams are as follows:

$$
\begin{aligned}
E_{\text {ref }}= & A_{\text {ref }} \sin \left(\omega_{\text {ref }} t+\phi_{0}+\phi_{\text {ref }}\right), \\
E_{\text {meas }}= & \sqrt{a} A_{\text {meas }} \cos \left(\omega_{\text {meas }} t+\phi_{0}+\phi_{\text {meas }, 1}\right) \\
& +\sqrt{b} A_{\text {meas }} \cos \left(\omega_{\text {meas }} t+\phi_{0}+\phi_{\text {meas } 2}\right),
\end{aligned}
$$

where $a^{2}$ and $b^{2}$ represent the fractions of the measurement spot reflected from the top and bottom trench surfaces respectively, such that $a^{2}+b^{2}=1$, and $A_{\text {ref }}$ and $A_{\text {meas }}$ are the electric field amplitudes of the reference and measurement beams, respectively. The ac portion of the intensity at the second photodetector is described by

$$
\begin{aligned}
I_{2, \mathrm{ac}} \propto & E_{\text {ref }} E_{\text {meas }} \\
\propto & \sin \left(\omega_{\text {ref }} t+\phi_{0}+\phi_{\text {ref }}\right)\left(\sqrt{a} \cos \left(\omega_{\text {meas }} t+\phi_{0}+\phi_{\text {meas }, 1}\right)\right. \\
& \left.+\sqrt{b} \cos \left(\omega_{\text {meas }} t+\phi_{0}+\phi_{\text {meas }, 2}\right)\right)
\end{aligned}
$$

such that the photodetector output, signal $\mathrm{BB}$, is

$$
\begin{aligned}
\mathrm{BB} \propto & \frac{\sqrt{a}}{2} \sin \left[\left(\omega_{\text {ref }}-\omega_{\text {meas }}\right) t+\left(\phi_{\text {ref }}-\phi_{\text {meas }, 1}\right)\right] \\
& +\frac{\sqrt{b}}{2} \sin \left[\left(\omega_{\text {ref }}-\omega_{\text {meas }}\right) t+\left(\phi_{\text {ref }}-\phi_{\text {meas }, 2}\right)\right] .
\end{aligned}
$$

This functionality results in a nearly linear relationship between the phase of signal BB from its value at the top surface to its value at the bottom surface during movement of the measurement spot from the top to the bottom trench surface as is depicted in Fig. 3. A nearly linear increase (decrease) in the phase angle of signal $\mathrm{BB} \phi_{\mathrm{BB}}\left(\phi_{m}\right)$, which can be closely approximated $\left(R^{2}=0.094\right)$ by the weighted average of the two phases $\phi_{\text {meas, } 1}$ and $\phi_{\text {meas,2 }}$ with respect to $\sqrt{a}$, the fraction of the electric field reflecting from the top surface, is therefore expected during spot movement from the top to the bottom trench surfaces. This phenomena is indeed present in the experimental data presented in Sec. III.

\section{Fractional fringe analysis: aluminum trench}

The first set of preliminary experiments performed involves scanning over a trench in a homogeneous layer of aluminum. In this scenario both the top and bottom trench surfaces are highly reflective and the highly opaque aluminum sufficiently thick that we need not concern ourselves with reflections from underlying material layers.

Figure 4 depicts the reflection of the measurement arm of the interferometer off of a reflective material at two successive positions in the raster scan: $x_{1}$ and $x_{2}$. Assuming any deviations of the reflection direction due to surface rough- 


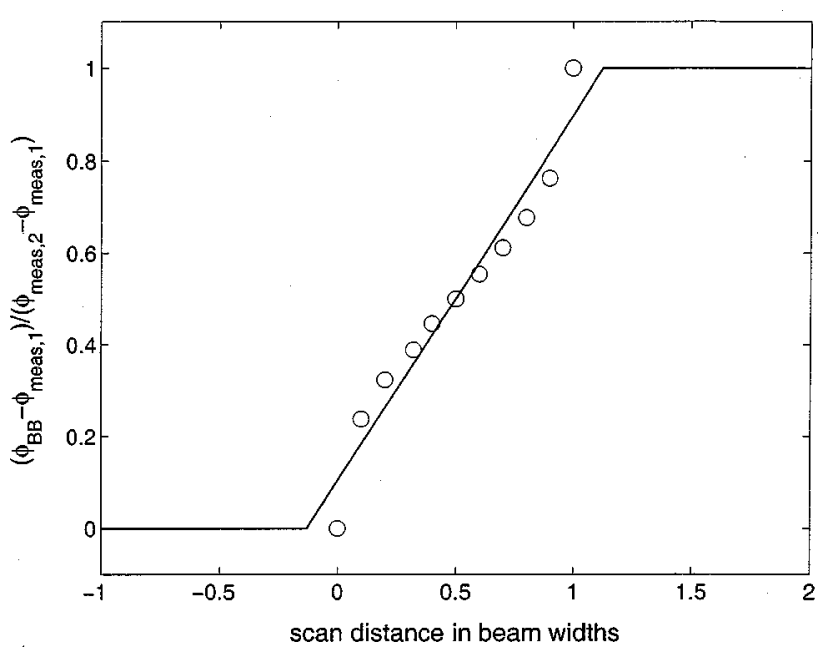

FIG. 3. Nearly linear response $\left(R^{2}=0.9996\right)$ of phase angle of signal BB $\left(\phi_{\mathrm{BB}}\right)$ during measurement spot movement from the top $(b=0)$ to the bottom $(b=1)$ trench surfaces.

ness to be negligible, we can take the angle of incidence $\phi_{0}$ to be zero. Then the total path lengths are $2 L$ at position $x_{1}$ and $2 L+2 \Delta L$ at position $x_{2}$. The phases of the reflected measurement arm signals $[\mathrm{Eq} .(10)]$ can therefore be expressed as

$$
\begin{aligned}
\phi_{\text {meas }, x_{1}} & =-\frac{4 \pi}{\lambda} L, \\
\phi_{\text {meas }, x_{2}} & =-\frac{4 \pi}{\lambda}(L+\Delta L),
\end{aligned}
$$

where $\lambda$ is the nominal wavelength of $632.8 \mathrm{~nm}$. The output signals $\mathrm{BB}$ from the second photodetector are as follows:

$$
\begin{aligned}
& \mathrm{BB}_{x_{1}} \propto \sin \left[\left(\omega_{\text {ref }}-\omega_{\text {meas }}\right) t+\left(\phi_{\text {ref }}+\frac{4 \pi}{\lambda} L-\phi_{1}\right)\right], \\
& \mathrm{BB}_{x_{2}} \propto \sin \left[\left(\omega_{\text {ref }}-\omega_{\text {meas }}\right) t+\left(\phi_{\text {ref }}+\frac{4 \pi}{\lambda}(L+\Delta L)-\phi_{1}\right)\right],
\end{aligned}
$$

where $\phi_{1}$ represents the phase change due to reflection at the aluminum surface

$$
\tan \phi_{i}=\frac{-2 k_{i} n_{i-1}}{n_{i}^{2}-n_{i-1}^{2}+k_{i}^{2}},
$$

where $n_{0}=1.0$ and $n_{1}=1.31$ are the refractive indices of air and aluminum, respectively, and $k_{1}=7.62$ is the extinction coefficient of aluminum. ${ }^{19}$

Thus, it can be seen that the phase of signal BB increases as $\Delta L$ increases. This can be expressed as follows:

$$
\begin{aligned}
& \phi_{\mathrm{BB}, x_{1}}=\phi_{\mathrm{ref}}+\frac{4 \pi}{\lambda} L-\phi_{1}, \\
& \phi_{\mathrm{BB}, x_{2}}=\phi_{\mathrm{ref}}+\frac{4 \pi}{\lambda}(L+\Delta L)-\phi_{1} .
\end{aligned}
$$

The change in phase of signal $\mathrm{BB}$ as a function of $\Delta L$ is therefore

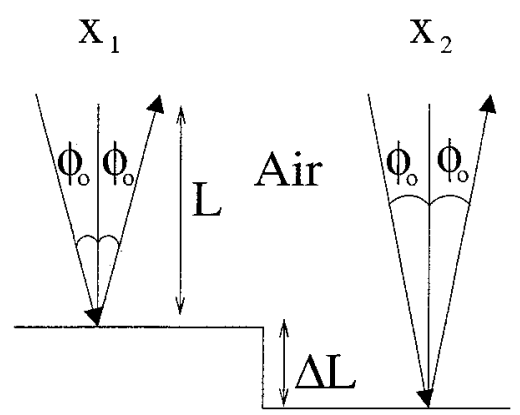

\section{Aluminum}

FIG. 4. Depiction of measurement beam reflection off of a reflective surface (aluminum) at two discrete positions: $x_{1}$ and $x_{2}$.

$$
\begin{aligned}
\Delta \phi_{\mathrm{BB}} & =\phi_{\mathrm{BB}, x_{2}}-\phi_{\mathrm{BB}, x_{1}} \\
& =\frac{4 \pi}{\lambda} \Delta L .
\end{aligned}
$$

Now, since our phase meter is measuring the phase of signal $\mathrm{AA}$ with respect to the phase of signal $\mathrm{BB}$, the change in measured phase is

$$
\begin{aligned}
\Delta \phi_{m} & =-\Delta \phi_{\mathrm{BB}} \\
& =-\frac{4 \pi}{\lambda} \Delta L \\
& =-\frac{4 \pi}{\lambda}(\Delta N+\Delta \epsilon),
\end{aligned}
$$

where $\Delta N$ is the change in integer fringe number and $\Delta \epsilon$ is the change in fractional fringe number. Since the phase measurement $\phi_{m}$ is modulo $2 \pi$ the phase measurement relates directly to the fractional fringe number

$$
\Delta \epsilon=-\frac{\lambda \Delta \phi_{m}}{4 \pi}
$$

and the integer fringe number needs to be determined via one of the methods discussed in Sec. II B.

\section{Fractional fringe analysis: photopatterned trench}

In contrast to the previous scenario in which the target surface is highly reflective and reflections from underlying material layers are negligible, the photoresist film is highly transmissive and reflections from underlying material layers must be accounted for during surface profiling of the photopatterned trench. Figure 5 depicts the wafer surface examined in this case study. The photoresist layer is highly transmissive while the underlying nickel-iron layer, like the aluminum layer of Sec. II C, is highly reflective and opaque. This leads to multiple reflections from each material layer at each point in the raster scan as depicted in Fig. 5.

Reflection and transmission coefficients of the electric fields are calculated as follows: ${ }^{20}$ 


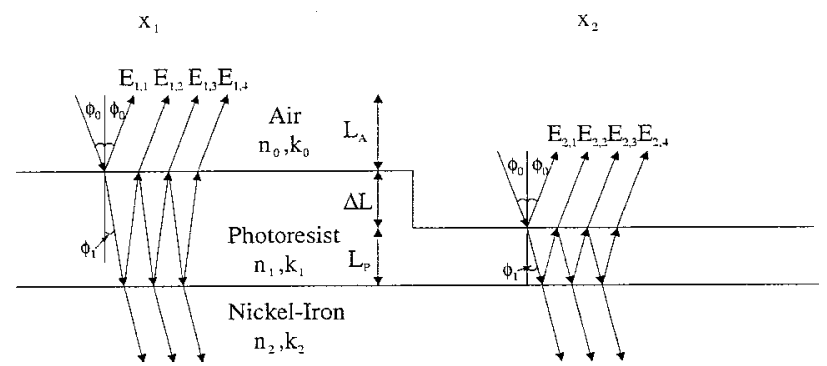

FIG. 5. Depiction of measurement beam reflection off of a transmissive surface (photoresist) at two discrete positions: $x_{1}$ and $x_{2}$.

$$
\begin{aligned}
& r_{1}=\sqrt{R_{1}}=\sqrt{\frac{\left(n_{0}-n_{1}\right)^{2}+\left(k_{0}-k_{1}\right)^{2}}{\left(n_{0}+n_{1}\right)^{2}+\left(k_{0}+k_{1}\right)^{2}},} \\
& r_{1}^{\prime}=\sqrt{R_{1}^{\prime}}=\sqrt{\frac{\left(n_{1}-n_{0}\right)^{2}+\left(k_{1}-k_{0}\right)^{2}}{\left(n_{1}+n_{0}\right)^{2}+\left(k_{1}+k_{0}\right)^{2}},} \\
& r_{2}=\sqrt{R_{2}}=\sqrt{\frac{\left(n_{1}-n_{2}\right)^{2}+\left(k_{1}-k_{2}\right)^{2}}{\left(n_{1}+n_{2}\right)^{2}+\left(k_{1}+k_{2}\right)^{2}},} \\
& t_{1}=\sqrt{T_{1}}=\sqrt{1-R_{1}}, \\
& t_{1}^{\prime}=\sqrt{T_{1}^{\prime}}=\sqrt{1-R_{1}^{\prime}},
\end{aligned}
$$

where $r_{i}$ and $t_{i}$ are the reflection and transmission coefficients for light propagation from layer $i-1$ to layer $i, r_{i}^{\prime}$ and $t_{i}^{\prime}$ are the reflection and transmission coefficients for propagation from layer $i$ to layer $i-1, n_{i}$ is the refractive index of layer $i$, and $k_{i}$ is the extinction coefficient of layer $i$.

Values for air at $632.8 \mathrm{~nm}$ are known to be approximately $n_{0}=1$ and $k_{0}=0$. Reported values for the photoresist (AZP4400 photoresist, AZ Electronics, Somerville, NJ) range linearly from $n_{1}=1.71$ at $365 \mathrm{~nm}$ to $n_{1}=1.67$ at 436 $\mathrm{nm}$. Extrapolating linearly to $632.8 \mathrm{~nm}$ yields $n_{1}=1.56$. Reported values for the extinction coefficients for the photoresist material are on the order of $0.001-0.01$ for the ultraviolet (UV) region, however the trend is not linear and no extrapolation can be made due to the visible region. For our purposes we employ a value of $k_{1}=0.001$ and therefore basically neglect absorbance in the photoresist layer. Reported values are not available for the nickel-iron permalloy which is $83 / 17 \mathrm{wt} \%$. Values of the refractive index are reported to be $n=2.00$ and $n=2.84$ for nickel and iron, respectively, so that a weighted average of $n_{2}=2.14$ is used in this study. ${ }^{19}$ Similarly, extinction coefficients are reported to be $k=3.69$ and $k=3.42$ for nickel and iron, respectively, so that a weighted average of $k_{2}=3.64$ is employed. ${ }^{19}$

Next, we assume perpendicular incidence of the measurement beam. The maximum slope expected in the photoresist layer translates to an elevation angle on the order of $1^{\circ}$, which corresponds to a path length change of less than $1 \mathrm{~nm}$, which is below the noise level of the system. Thus, no significant system accuracy is lost by assuming the angle of incidence, $\phi_{0}$, and thus $\phi_{1}$, to be zero. Handling of the discontinuity of the photoresist side wall is discussed in Sec. III B.

We can now write the overall reflected amplitude as an infinite series of reflection and transmission terms ${ }^{20}$

$$
\begin{aligned}
R= & r_{1} e^{-i \delta_{1}}+t_{1} t_{1}^{\prime} r_{2} e^{-i \delta_{2}}+t_{1} t_{1}^{\prime} r_{1}^{\prime} r_{2}^{2} e^{-i \delta_{3}} \\
& +t_{1} t_{1}^{\prime} r_{1}^{\prime 2} r_{2}^{3} e^{-i \delta_{4}}+\cdots
\end{aligned}
$$

where $\delta_{i}$ is a phase change due to the path length traveled by each of the reflected beams.

We can now write the electric fields of the reflected beams at $x_{1}$ and $x_{2}$ as depicted in Fig. 5:

$$
\begin{aligned}
E_{\text {meas }, x_{1}}= & r_{1} A_{\text {meas }, 1} \cos \left(\omega_{\text {meas }} t-\frac{4 \pi L_{A}}{\lambda_{A}}+\phi_{0}+\phi_{1}\right)+t_{1} t_{1}^{\prime} r_{2} A_{\text {meas }, 1} \cos \left(\omega_{\text {meas }} t-\frac{4 \pi L_{A}}{\lambda_{A}}-\frac{4 \pi\left(L_{P}+\Delta L\right)}{\lambda_{P}}+\phi_{0}+\phi_{2}\right) \\
& +t_{1} t_{1}^{\prime} r_{1}^{\prime} r_{2}^{2} A_{\text {meas }, 1} \cos \left(\omega_{\text {meas }} t-\frac{4 \pi L_{A}}{\lambda_{A}}-\frac{8 \pi\left(L_{P}+\Delta L\right)}{\lambda_{P}}+\phi_{0}+\phi_{1}+2 \phi_{2}\right) \\
& +t_{1} t_{1}^{\prime} r_{1}^{\prime 2} r_{2}^{3} A_{\text {meas }, 1} \cos \left(\omega_{\text {meas }} t-\frac{4 \pi L_{A}}{\lambda_{A}}-\frac{12 \pi\left(L_{P}+\Delta L\right)}{\lambda_{P}}+\phi_{0}+2 \phi_{1}+3 \phi_{2}\right)+\cdots, \\
E_{\text {meas }, x_{2}=} & r_{1} A_{\text {meas }, 2} \cos \left(\omega_{\text {meas }} t-\frac{4 \pi\left(L_{A}+\Delta L\right)}{\lambda_{A}}+\phi_{0}+\phi_{1}\right)+t_{1} t_{1}^{\prime} r_{2} A_{\text {meas }, 2} \cos \left(\omega_{\text {meas }} t-\frac{4 \pi\left(L_{A}+\Delta L\right)}{\lambda_{A}}-\frac{4 \pi L_{P}}{\lambda_{P}}+\phi_{0}+\phi_{2}\right) \\
& +t_{1} t_{1}^{\prime} r_{1}^{\prime} r_{2}^{2} A_{\text {meas }, 2} \cos \left(\omega_{\text {meas }} t-\frac{4 \pi\left(L_{A}+\Delta L\right)}{\lambda_{A}}-\frac{4 \pi L_{P}}{\lambda_{P}}+\phi_{0}+\phi_{1}+2 \phi_{2}\right) \\
& +t_{1} t_{1}^{\prime} r_{1}^{\prime 2} r_{2}^{3} A_{\text {meas }, 2} \cos \left(\omega_{\text {meas }} t-\frac{8 \pi\left(L_{A}+\Delta L\right)}{\lambda_{A}}-\frac{12 \pi L_{P}}{\lambda_{P}}+\phi_{0}+2 \phi_{1}+2 \phi_{2}\right)+\cdots,
\end{aligned}
$$


where $\phi_{0}$ is the phase change due to target vibration and $\phi_{1}$ and $\phi_{2}$ are phase changes due to reflection off the photoresist and nickel-iron layers, respectively, and are given by $^{21}$

$$
\tan \phi_{i}=\frac{-2 k_{i} n_{i-1}}{n_{i}^{2}-n_{i-1}^{2}+k_{i}^{2}} .
$$

The output signals BB from the second photodetector are therefore as follows:

$$
\begin{aligned}
\mathrm{BB}_{x_{1}} \propto & r_{1} \sin \left[\left(\omega_{\text {ref }}-\omega_{\text {meas }}\right) t+\left(\phi_{\text {ref }}+\frac{4 \pi L_{A}}{\lambda_{A}}-\phi_{1}\right)\right] \\
& +t_{1} t_{1}^{\prime} r_{2} \sin \left[\left(\omega_{\text {ref }}-\omega_{\text {meas }}\right) t\right. \\
& \left.+\left(\phi_{\text {ref }}+\frac{4 \pi L_{A}}{\lambda_{A}}+\frac{4 \pi\left(L_{P}+\Delta L\right)}{\lambda_{P}}-\phi_{2}\right)\right]+\cdots, \quad(60) \\
\mathrm{BB}_{x_{2}} \propto r_{1} \sin \left[\left(\omega_{\text {ref }}-\omega_{\text {meas }}\right) t+\left(\phi_{\text {ref }}+\frac{4 \pi\left(L_{A}+\Delta L\right)}{\lambda_{A}}-\phi_{1}\right)\right] & \\
& +t_{1} t_{1}^{\prime} r_{2} \sin \left[\left(\omega_{\text {ref }}-\omega_{\text {meas }}\right) t\right. \\
& \left.+\left(\phi_{\text {ref }}+\frac{4 \pi\left(L_{A}+\Delta L\right)}{\lambda_{A}}+\frac{4 \pi L_{P}}{\lambda_{P}}-\phi_{2}\right)\right]+\cdots
\end{aligned}
$$

Using the linear approximation for the addition of phases as described in Sec. II B, the phase of the output signals BB can be expressed as

$$
\begin{aligned}
\phi_{\mathrm{BB}, x_{1}}= & \frac{r_{1}}{D}\left(\phi_{\mathrm{ref}}+\frac{4 \pi L_{A}}{\lambda_{A}}-\phi_{1}\right)+\frac{t_{1} t_{1}^{\prime} r_{2}}{D}\left(\phi_{\mathrm{ref}}+\frac{4 \pi L_{A}}{\lambda_{A}}\right. \\
& \left.+\frac{4 \pi\left(L_{P}+\Delta L\right)}{\lambda_{P}}-\phi_{2}\right)+\cdots, \\
\phi_{\mathrm{BB}, x_{2}}= & \frac{r_{1}}{D}\left(\phi_{\mathrm{ref}}+\frac{4 \pi\left(L_{A}+\Delta L\right)}{\lambda_{A}}-\phi_{1}\right)+\frac{t_{1} t_{1}^{\prime} r_{2}}{D}\left(\phi_{\mathrm{ref}}\right. \\
& \left.+\frac{4 \pi\left(L_{A}+\Delta L\right)}{\lambda_{A}}+\frac{4 \pi L_{P}}{\lambda_{P}}-\phi_{2}\right)+\cdots,
\end{aligned}
$$

where

$$
\begin{aligned}
D & =r_{1}+t_{1} t_{1}^{\prime} r_{2}\left(1+r_{1}^{\prime} r_{2}+r_{1}^{\prime 2} r_{2}^{2}+\cdots\right) \\
& =r_{1}+\frac{t_{1} t_{1}^{\prime} r_{2}}{1-r_{1}^{\prime} r_{2}} .
\end{aligned}
$$

The change in phase of signal $\mathrm{BB}$ as a function of $\Delta L$ is therefore

$$
\begin{aligned}
\Delta \phi_{\mathrm{BB}}= & \phi_{\mathrm{BB}, x_{2}}-\phi_{\mathrm{BB}, x_{1}} \\
\approx & \frac{4 \pi r_{1} \Delta L}{\lambda_{A} D}+\frac{4 \pi t_{1} t_{1}^{\prime} r_{2} \Delta L}{D}\left(\frac{1}{\lambda_{A}}-\frac{1}{\lambda_{P}}\right) \\
& \times\left(1+2 r_{1}^{\prime} r_{2}+3 r_{1}^{\prime 2} r_{2}^{2}+\cdots\right) \\
\approx & \frac{4 \pi r_{1} \Delta L}{\lambda_{A} D}+\frac{4 \pi t_{1} t_{1}^{\prime} r_{2} \Delta L}{D}\left(\frac{1}{\lambda_{A}}-\frac{1}{\lambda_{P}}\right)\left(\frac{1}{\left(1-r_{1}^{\prime} r_{2}\right)^{2}}\right) .
\end{aligned}
$$

Now, since the phase meter is measuring the phase of signal AA with respect to signal $\mathrm{BB}$, the change in measured phase is

$$
\begin{aligned}
\Delta \phi_{m} & =-\Delta \phi_{\mathrm{BB}} \\
& \approx \frac{-4 \pi r_{1} \Delta L}{\lambda_{A} D}+\frac{4 \pi t_{1} t_{1}^{\prime} r_{2} \Delta L}{D}\left(\frac{1}{\lambda_{P}}-\frac{1}{\lambda_{A}}\right)\left(\frac{1}{\left(1-r_{1}^{\prime} r_{2}\right)^{2}}\right) .
\end{aligned}
$$

As before, since the phase measurement is modulo $2 \pi$ the phase measurement relates directly to the fractional fringe number

$$
\Delta \epsilon \approx \frac{\Delta \phi_{m}}{\frac{-4 \pi r_{1}}{\lambda_{A} D}+\frac{4 \pi t_{1} t_{1}^{\prime} r_{2}}{D}\left(\frac{1}{\lambda_{P}}-\frac{1}{\lambda_{A}}\right)\left(\frac{1}{\left(1-r_{1}^{\prime} r_{2}\right)^{2}}\right)},
$$

where $\Delta \varepsilon$ is the fractional fringe number and is the difference in height between positions $x_{1}$ and $x_{2}$ assuming that there is no change in the integer fringe number (i.e., $\Delta L$ $=\Delta \varepsilon$ ). Notice that for the case of the photoresist an increase in measured phase corresponds to a decrease in surface height since the second term in the denominator outweighs the first term due to the fact that $\left[\left(1 / \lambda_{P}\right)-\left(1 / \lambda_{A}\right)\right]>0$. This is the inverse of the situation for purely reflective materials such as the aluminum film discussed in Sec. II C.

\section{RESULTS AND DISCUSSION}

\section{A. Aluminum trench}

An interferometric surface scan across a trench in a homogeneous layer of aluminum was repeated ten times. A cross-sectional depiction of the substrate investigated in this case study is found in Fig. 4. Scanning was performed by mounting the wafer on a computer-controlled, single-axis translation stage, which moves the wafer in the $x$ direction to facilitate the movement of the measurement and reference arms across the contours of the wafer surface. Raster scanning was accomplished through the use of a uniaxial translation stage which is controlled by a Unidex 11 (Aerotech, Inc, Pittsburgh, PA) controller connected via general purpose interface bus (GPIB) to a PC running LABVIEW (National Instruments, Austin, TX) data acquisition software.

Optimal focus of the system was achieved through preliminary experiments in which the slope of the phase measurement as the measurement beam moved into the trench was monitored as a function of focal distance. Examination of Eq. (37) shows that minimum spot size is found at maximum values of this slope. The focal depth of the lens system was approximately $500 \mu \mathrm{m}$ so that an area of $y=0$ $\pm 500 \mu \mathrm{m}$ was probed during the search for the maximum slope, where $y=0$ corresponds to the initial focal length which was set by maximizing the amplitude of the heterodyne return signal BB. Figure 6 contains a plot of slope values as a function of the $y$ position. It can be seen that the maximum slope, and thus the smallest spot size, was found at $y=+200 \mu \mathrm{m}$. This focal distance was therefore employed in the ten experiments described herein.

It was observed that phase jitter attributed to the movement of the wafer surface during scanning is nonexistent due 


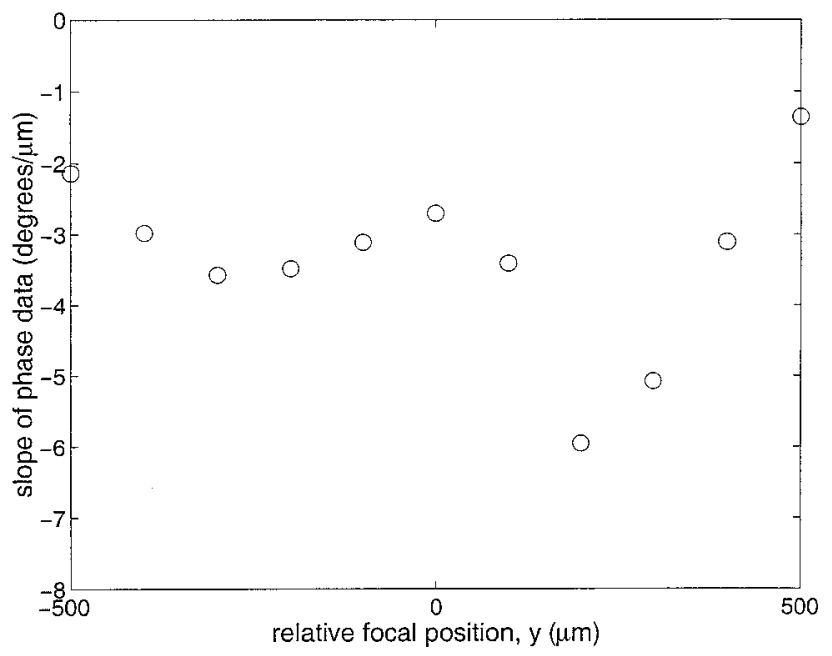

FIG. 6. Slope of phase into trench as a function of the $y$ position of the wafer surface. Maximum slope (optimal focal point) is found at $y=$ $+200 \mu \mathrm{m}$.

to the common-path design of the interferometer. However, the portion of the interferometer where the reference and measurement arms are not coaxial (i.e., between the polarizing beam splitter cube and the last reference arm mirror of Fig. 1) was found to be sensitive to ambient air currents and temperature conditions. To lessen the sensitivity of the interferometer to environmental conditions, this portion of the interferometer was enclosed in a plexi-glass shield and the interferometer was allowed to reach a thermal equilibrium before beginning measurement runs.

The translation stage was moved in increments of $1 \mu \mathrm{m}$. It should be noted that since the measurement beam is approximately $8 \mu \mathrm{m}$ in diameter $\left(w_{0}=4 \mu \mathrm{m}\right)$ the scanning technique produced a moving average over the surface roughness. This had no negative effect on the determination of trench depth, which was taken to be the average difference in height between the top and bottom surfaces of the trench, and allowed a more exact determination of the trench width due to the small step size of the scan.

Ten phase measurements were taken at each stage position. The average and standard deviation of these measurements were recorded on the PC which was interfaced with the translation stage controller and the phase meter via GPIB connections. The number of phase measurements performed at each step was varied in some experiments, but analysis of variance testing suggested that there was no correlation between the number of measurements and the standard deviation of the sample population. The overall mean standard deviation of the phase measurements for the set of experiments was found to be $3.5^{\circ}$, which corresponds to a standard deviation of $3.1 \mathrm{~nm}$ in surface height due to measurement noise.

The raw data were collected and a plane-fitting procedure was performed, during which a linear line was fit to and subsequently subtracted from the data set, thereby removing any phase change associated with nonperpendicular alignment of the wafer surface. Figure 7 depicts a representative example of a raw data set. Notice the linear decrease (increase) in $\phi_{m}$ as the measurement beam moves into (out of)

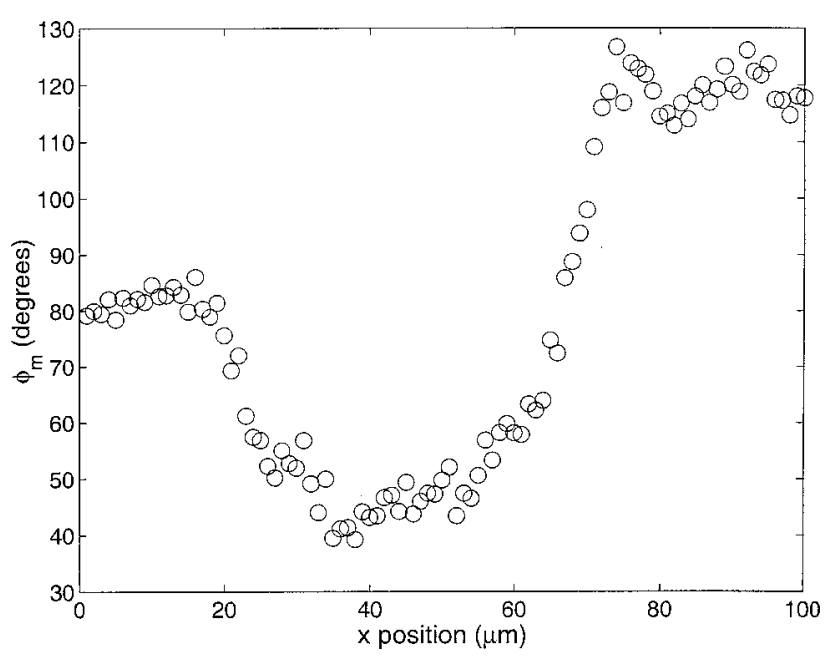

FIG. 7. Representative data set of $\phi_{m}$ vs position collected while scanning a trench in a homogeneous layer of aluminum.

the trench, as predicted by Eq. (37). Notice also the linear increase in $\phi_{m}$ across the entire data set, which can be attributed to nonpreciseness in the alignment of the wafer surface. Removing this linear trend results in the conditioned data set depicted in Fig. 8. This data set is then converted to surface height by applying Eqs. (49) and (50). A priori knowledge of the approximate trench height allows us to set $\Delta N$ of Eq. (49) to 3.

The slopes into and out of the trench were accounted for as follows in the data analysis. The starting and stopping points of the two slopes were identified and average values of $\phi_{m}$ were calculated for both the top and bottom surfaces of the trench. Points lying on the downward slope were assigned the average bottom surface $\phi_{m}$ value and points lying on the upward slope were assigned the average top surface $\phi_{m}$ value. Thus we are assuming that the beginning of the downward slope signifies the first portion of the measurement beam falling into the trench, and that the subsequent beginning of the upward slope signifies this same portion of the measurement beam climbing out of the trench to the top surface of the material layer. Figure 9 contains the final sur-

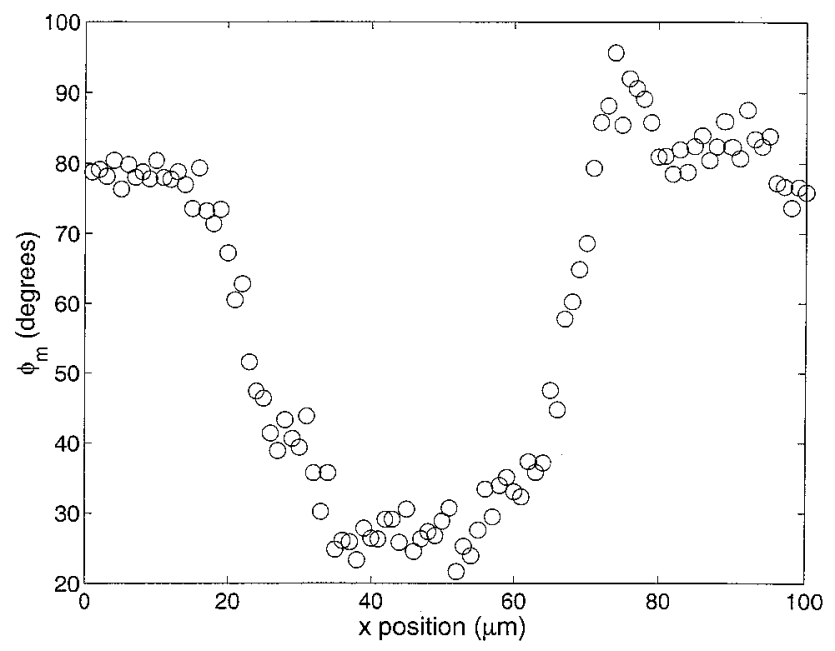

FIG. 8. The data set of Fig. 7 upon removing the linear trend due to imperfect alignment of the wafer surface. 


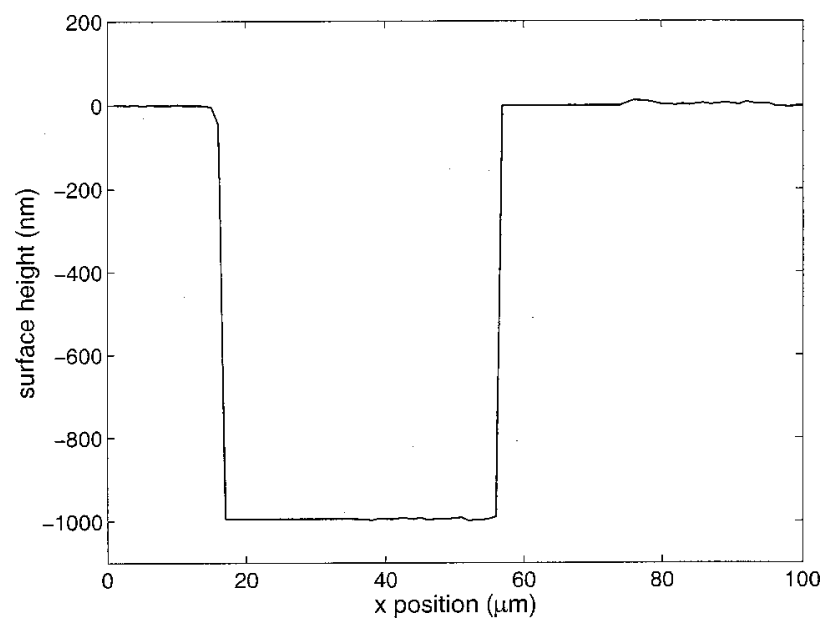

FIG. 9. Surface profile determined by converting the conditioned data set of Fig. 8 to units of surface height.

face profile obtained upon converting the phase data to surface height information.

The average standard deviation of surface height over flat portions of the surface was found to be $4.6 \mathrm{~nm}$, of which $3.1 \mathrm{~nm}$ (the mean standard deviation of the phase measurements) is attributed to measurement noise and the remainder is attributed to surface roughness. This $4.6 \mathrm{~nm}$ standard deviation in surface height measurement leads to a standard deviation of $6.5 \mathrm{~nm}$ in trench depth since the $4.6 \mathrm{~nm}$ uncertainty is present in both the top and bottom surfaces of the trench. The 95\% confidence interval on trench depth is therefore the average measured depth $\pm 13.0 \mathrm{~nm}$.

Histograms of the measured trench widths and depths are shown in Figs. 10 and 11. The average trench width and depth of the ten experiments were found to be $41.1 \mu \mathrm{m}$ and $991.6 \mathrm{~nm}$ with standard deviations of $1.9 \mu \mathrm{m}$ and $4.4 \mathrm{~nm}$, respectively. Notice that the standard deviation in the observed trench depth of $4.4 \mathrm{~nm}$ is well within the expected standard deviation of $6.5 \mathrm{~nm}$.

These results are in good agreement with surface profiles of the same trench determined using both a stylus-based

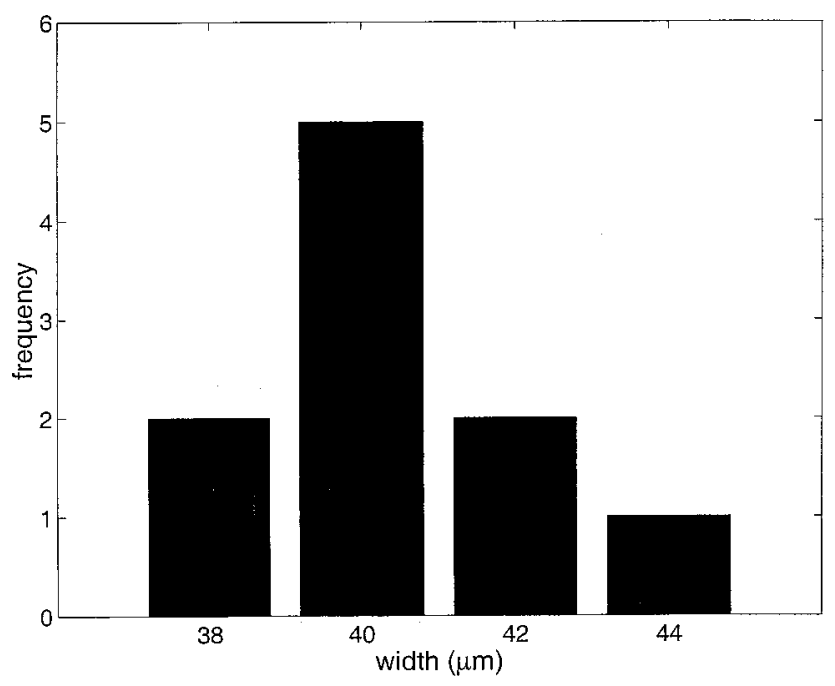

FIG. 10. Trench width histogram, average $=41.1 \mu \mathrm{m}$, standard deviation $=1.9 \mu \mathrm{m}$.

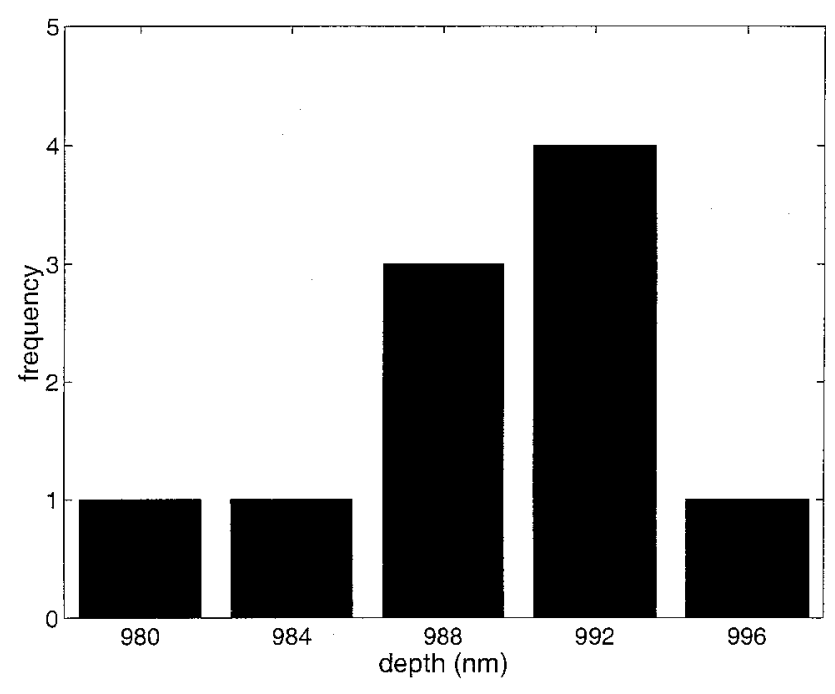

FIG. 11. Trench depth histogram, average $=991.6 \mathrm{~nm}$, standard deviation $=4.4 \mathrm{~nm}$.

scanning profilometer (Alpha-Step, KLA Tencor, San Jose, CA) and an atomic force microscope (Nanoscope SPM, Digital Instruments, Santa Barbara, CA). The average trench width as measured by the stylus scanner is $44.4 \mu \mathrm{m}$ which is in agreement with the interferometric measurement of 41.1 $\mu \mathrm{m}$. The inaccuracies in exact trench width are due to differences in the cross-sectional areas of the measurement beam and the stylus. The $95 \%$ confidence interval on trench depth as measured by the stylus scanner is $959 \pm 50 \mathrm{~nm}$. That measured by the atomic force microscope (AFM) is 1034.7 $\pm 57.8 \mathrm{~nm}$, where the $95 \%$ confidence interval is due to an average standard deviation of $20.4 \mathrm{~nm}$ in surface height measurement due to measurement noise and surface roughness.

Figure 12 contains a plot of the $95 \%$ confidence interval for trench depth measurement for each of the three measurement devices. Notice that the uncertainties associated with each of the three measurement devices lead to the overlapping of the 95\% confidence intervals on trench depth, thus indicating that there is no statistical difference between the

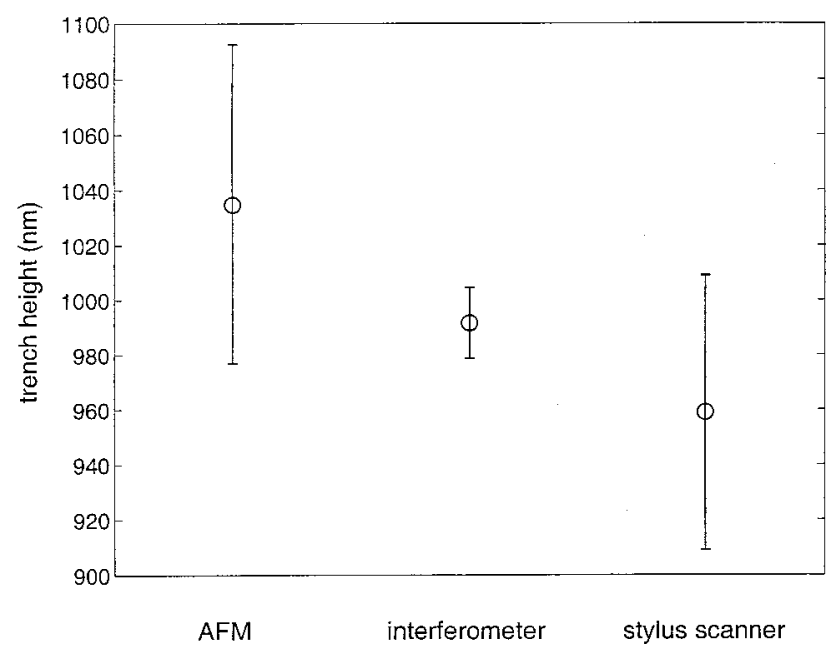

FIG. 12. Comparison of trench height as determined by the interferometric scanner, atomic force microscopy, and a stylus-based scanner. 


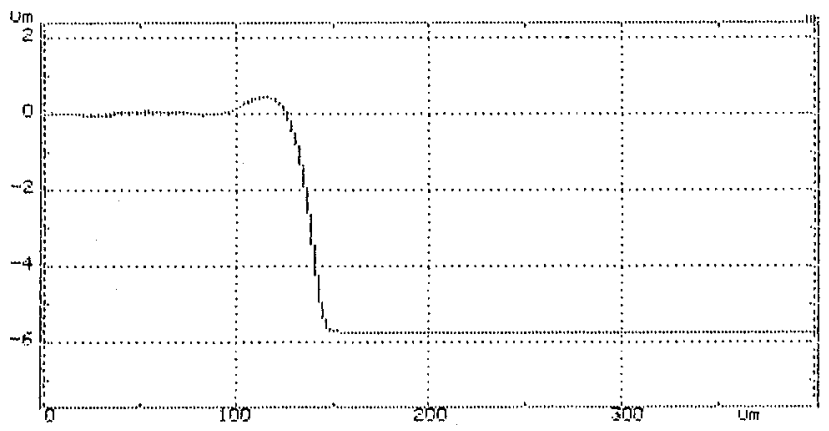

FIG. 13. Surface profile of a photopatterned trench wall obtained through the use of a stylus-based scanner.

trench depths as measured by each of the three devices. Finally, notice that the $95 \%$ confidence interval of the interferometric trench depth measurement is more than fivefold smaller than the confidence intervals associated with the AFM and stylus measurements. This indicates that there is significantly less uncertainty in the interferometric measurements and that the laser interferometer developed in this work is therefore the more accurate and repeatable of the three measurement techniques.

\section{B. Photopatterned trench}

The interferometric surface profiler was further tested by scanning across a photopatterned 83/17 wt \% nickel-iron permalloy trench. A cross-sectional depiction of the substrate investigated in this case study is found in Fig. 5. This case study is a good example of the foreseen end use of the instrument: that is, scanning across a photopatterned trench to determine initial and in situ surface profiles to facilitate the online control of ion milling etch depth.

Figure 13 contains a surface profile of a photopatterned trench wall obtained with the Tencor stylus scanner. Notice the curvature at the trench wall corner which is due to the postbake step of the photolithography process. Scans were performed both over a single photopatterned wall as is depicted in Fig. 13 and over an entire photopatterned trench.

Figure 14 shows typical sets of raw phase and intensity data generated by scanning over a single wall similar to that depicted in Fig. 13. The raw phase data can be explained as follows. Starting from the leftmost end of the data set $(x$ $=0 \mu \mathrm{m}$ ), the sharp downward slope in $\phi_{m}$ which begins at $x \approx 25 \mu \mathrm{m}$ corresponds to the beginning of the upward slope which forms the curved wall corner in the photoreist layer. The subsequent upward slope in $\phi_{m}$, which begins at $x$ $\approx 55 \mu \mathrm{m}$, corresponds to the beginning of the downward slope in the photoresist after reaching the apex of the curved corner. Note that in the transmissive photoresist layer the measurement arm length is directly proportional to the measured phase, whereas with the reflective aluminum material of the previous section this dependency was inversely proportional. The change in the direction of the slope in $\phi_{m}$ at $x \approx 70 \mu \mathrm{m}$ corresponds to the nearly linear decrease in phase [predicted by Eq. (37)] as the measurement beam travels from the top of the photoresist layer to the bottom of the trench which is composed of highly reflective nickel-iron.
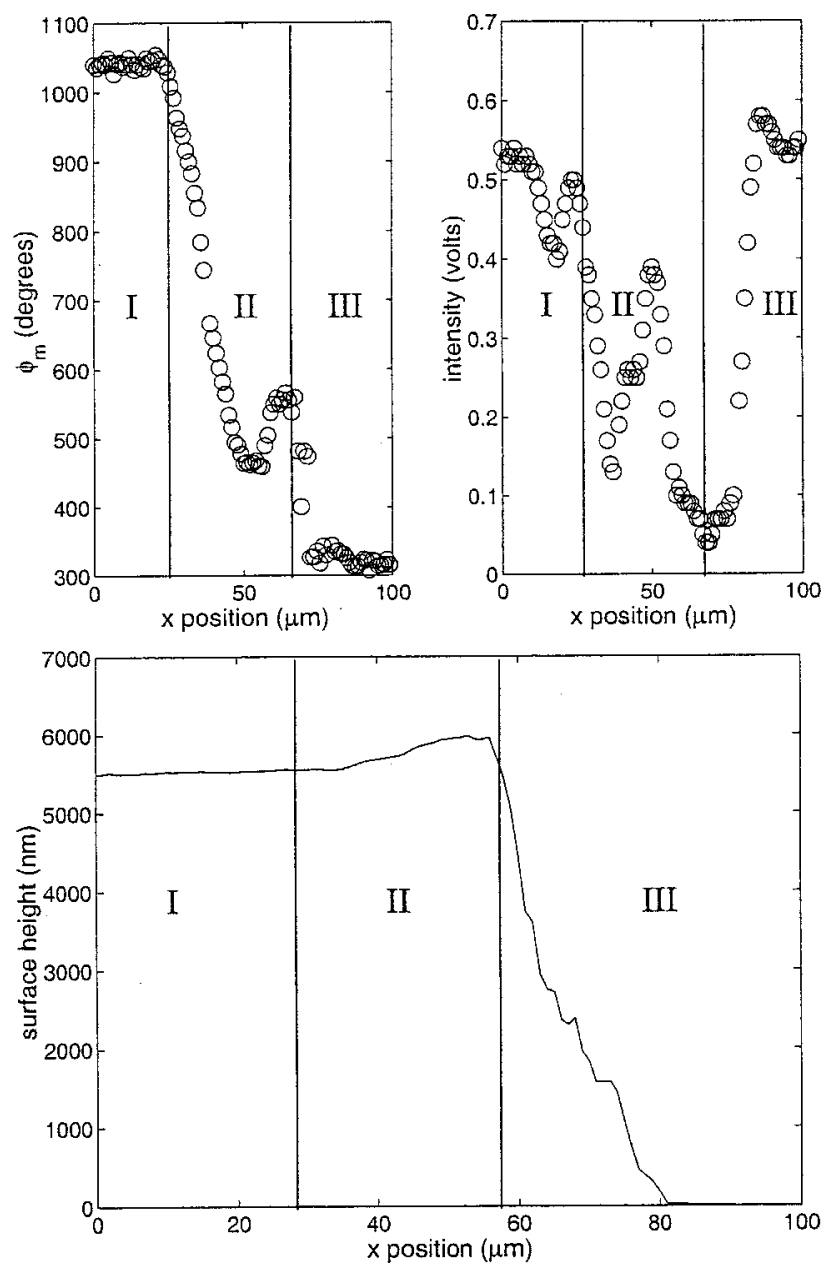

FIG. 14. Typical sets of $\phi_{m}$ and intensity data (top) collected during interferometric surface profiling of a photopatterned trench wall (bottom).

The intensity data show significant drops in signal strength at surface portions which exhibit extreme curvature (i.e., the trench wall and the curved trench corner). This is indicative of the fact that the portion of the measurement beam reflected from the top surface of the photoresist is significantly deviated upon reflection from these curved surfaces. The path length of the transmitted portion is, however, relatively unaffected. Path length changes due to refraction of the incident beam are on the order of $1 \mathrm{~nm}$ at the curved photoresist corner, which is significantly less than measurement noise levels and is therefore neglected.

Notice that the plots in Fig. 14 have been divided into three zones. Zone I corresponds to the transmissive photoresist layer and phase information is converted to surface height in this zone using Eq. (71). Zone III corresponds to the reflective nickel-iron layer and phase information in this zone is converted to surface height following the method detailed in Sec. II C.

Zone II contains the portion of the surface profile in which the surface exhibits significant curvature such that the beam reflected from the top surface of the photoresist is significantly deviated and is ignored in the calculations. This assumption is valid given the large working distance of the measurement arm of the interferometer which causes small perturbations in the angle of the return beam to lead to large 
deviations of the beam from the nominal beam path. Equation (71) then reduces to

$$
\Delta \epsilon=\frac{\Delta \phi_{m}}{\frac{4 \pi t_{1} t_{1}^{\prime} r_{2}}{E}\left(\frac{1}{\lambda_{P}}-\frac{1}{\lambda_{A}}\right)\left(\frac{1}{\left(1-r_{1}^{\prime} r_{2}\right)^{2}}\right)},
$$

where

$$
E=\frac{t_{1} t_{1}^{\prime} r_{2}}{1-r_{1}^{\prime} r_{2}}
$$

for this section of the surface profile.

Unlike the walls of the aluminum trench investigated in Sec. III A, the trench walls of the photopatterned part are not vertical and exhibit curvature as is shown in Fig. 13. This results in an ambiguity in the analysis of the phase data since the downward trend in the phase which begins at $x$ $\approx 70 \mu \mathrm{m}$ is due both to the beam being divided between the top and bottom surfaces of the trench and the movement of the measurement beam down the slope of the trench wall. This ambiguity was dealt with by spacing the points on this slope proportionately between the top and bottom surfaces of the trench during data analysis. That is,

$$
\phi_{\text {wall }}=\frac{\phi_{m}-\phi_{\text {bot }}}{\phi_{\text {top }}-\phi_{\text {bot }}}\left(\phi_{\text {top }}+18\left(360^{\circ}\right)-\phi_{1}+\phi_{2}-\phi_{\text {bot }}\right)+\phi_{\text {bot }} \text {, }
$$

where $\phi_{\text {wall }}$ refers to phase data which lie on this ambiguous slope, $\phi_{\text {bot }}$ refers to the phase at the trench floor, and $\phi_{\text {top }}$ refers to the phase at the top of the trench wall. Here $\phi_{1}$ and $\phi_{2}$ refer to the phase change upon reflection at the photoresist/air and nickel-iron/air interfaces, respectively. These values are calculated to be $\phi_{1}=-0.1^{\circ}$ and $\phi_{2}=$ $-23.4^{\circ}$ through the use of Eq. (42). The addition of 18 $\times 360^{\circ}$ to the phase at the top of the trench wall comes from the a priori knowledge of trench height as determined by the Tencor stylus scanner. This ambiguity decreases our knowledge of the exact surface profile of the trench wall, but it was shown that this approximation of the surface profile closely matches that obtained via the stylus scanner.

Phase data were converted to surface height information using Eqs. (71) and (72) and the a priori knowledge of $\Delta N$ $=18$, which was obtained from the results of the Tencor scan. A value of 0.63 was used for the reflectance of the nickel-iron layer $\left(R_{N}\right) .{ }^{19}$ The values of $\lambda_{P}$ and $R_{P}$ of Eq. (71) are highly dependent on the refractive index of the photoresist layer, $n_{P}$, which is not well known for visible wavelengths of light. It was therefore necessary to perform a calibration whereby the value of $n_{P}$ was adjusted until the height of the curved trench corner matched that which was expected.

Due to the fact that this trench corner height obtained via the Tencor stylus scanner is relatively inaccurate because of the low accuracy of the stylus-based instrument, a thin but opaque $(\approx 500 \AA)$ layer of aluminum was evaporated onto the photopatterned surface. Interferometric scans were performed across this aluminum coated surface and the phase data were analyzed using Eq. (50), which is independent of refractive index due to the high reflectivity of the aluminum

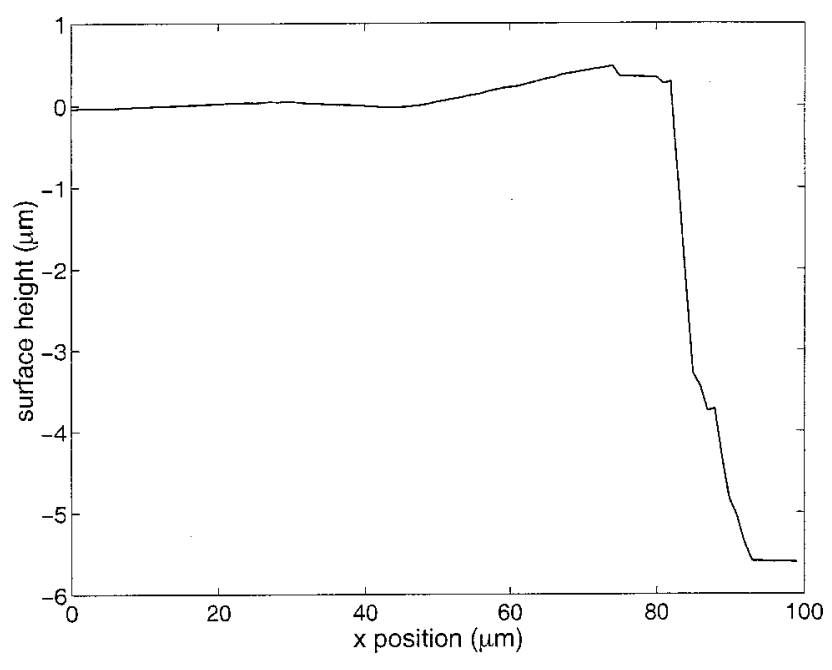

FIG. 15. Interferometric surface profile of an aluminum coated trench wall.

coating. Figure 15 contains an example of a wall profile which was obtained by scanning across this aluminum coated surface.

The average hill height as determined by a series of scans across the aluminum coated surface was determined to be $463.7 \pm 45.4 \mathrm{~nm}$, which corresponds well to that measured by the Tencor scanner (Fig. 13). Figure 16 contains plots of two interferometric scans across the photopatterned trench wall. The height of the hill at the top corner of the photoresist wall was matched to $463.7 \mathrm{~nm}$ by manipulating the refractive index value $n_{P}$, which was found to be 1.58 and 1.67 for the two scans, respectively. These values agree ac-
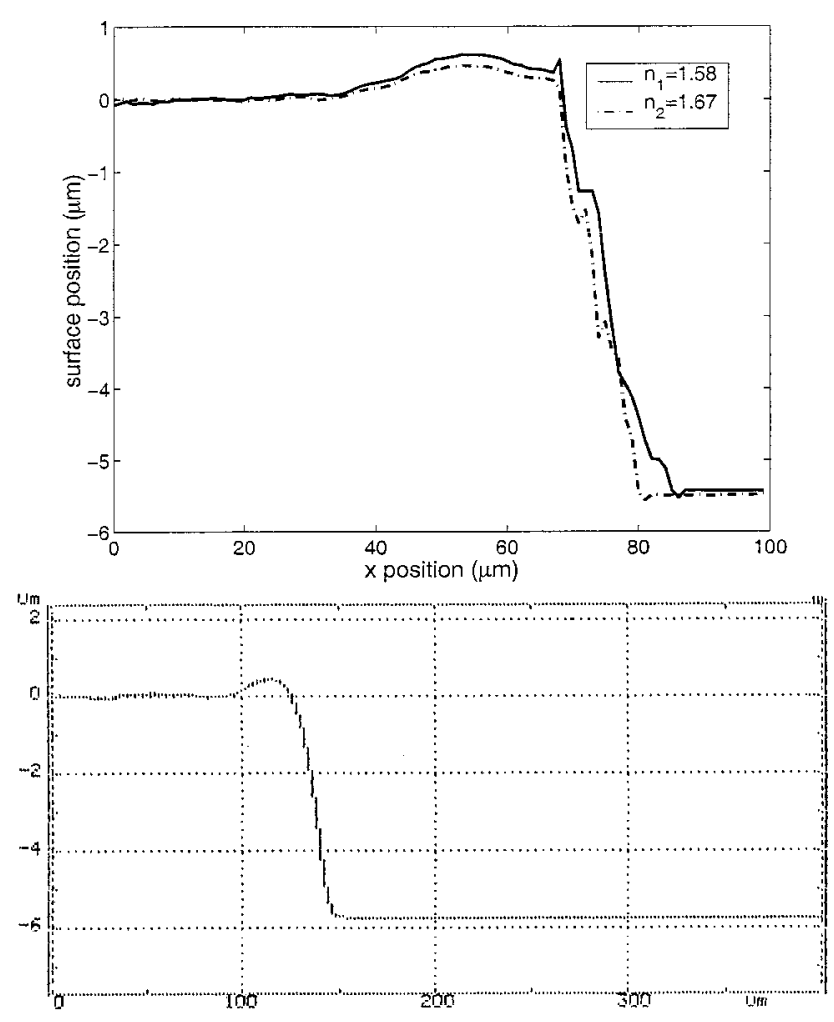

FIG. 16. Interferometric (top) and stylus-based (bottom) surface profiles of a photopatterned trench wall. 

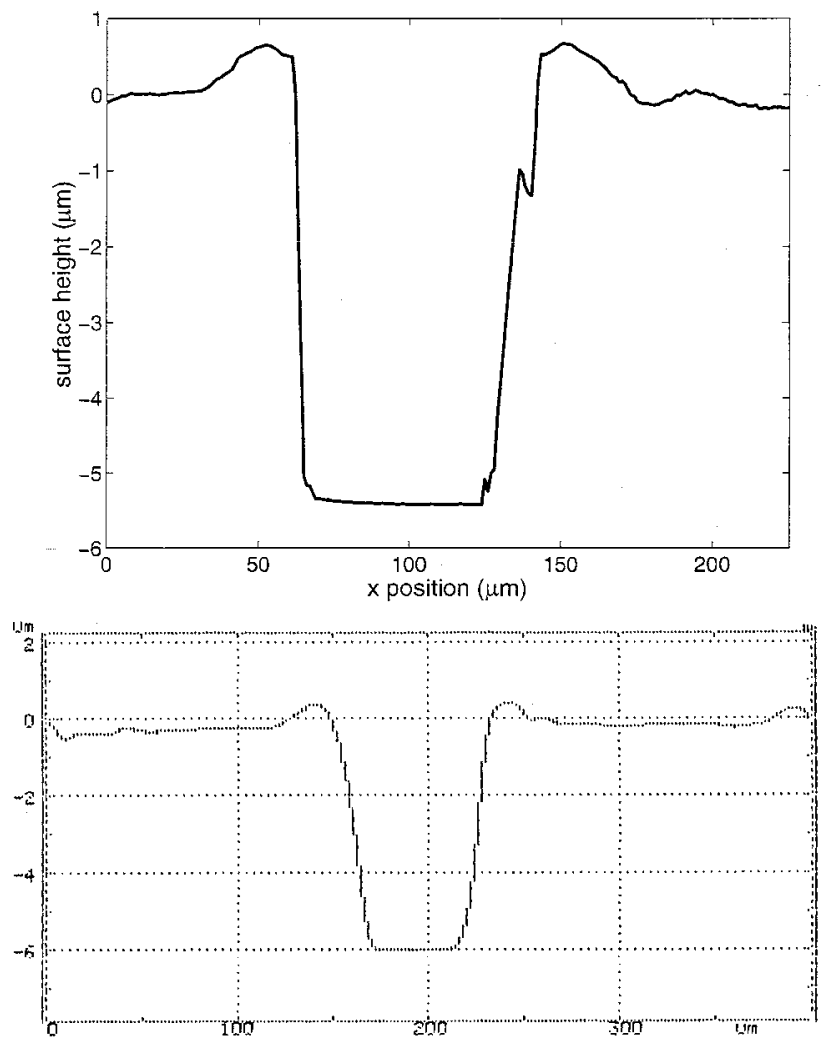

FIG. 17. Interferometric (top) and stylus-based (bottom) surface profiles of an entire photopatterned trench.

ceptably with the value of 1.56 , which was extrapolated from the UV range data in Sec. II D.

Finally, an interferometric scan was performed across an entire photopatterned trench and the average value of $n_{P}$ from the two photoresist wall scans, 1.63 , was used in the data reduction. Figure 17 contains the results of this analysis compared with the results of a Tencor stylus measurement of the same surface profile. It can be seen by examining Fig. 17 that the results of the surface profile obtained via an interferometric scan closely correspond to that obtained through the use of the stylus-based device. Trench depth and width as determined by a prototype common-path interferometer are shown to compare well with those measured with both an AFM and a stylus-based scanning device. It is further shown that the level of uncertainty associated with the interferometry scanner is significantly less than that of both the AFM and stylus scanning devices.

\section{ACKNOWLEDGMENTS}

This work has been partially funded by StorageTek (Louisville, CO). The authors would further like to thank Dr. Scott Diddams of JILA, University of Colorado (Boulder, $\mathrm{CO})$ for participating in useful discussions during the trouble-shooting phase of this work.

${ }^{1}$ S. Davies and B. Khamsehpour, J. Vac. Sci. Technol. B 11, 263 (1993).

${ }^{2}$ L. Harriott and J. Vasile, J. Vac. Sci. Technol. B 7, 181 (1989).

${ }^{3}$ R. Humphreys, N. Chew, S. Morgan, J. Satchell, and A. Cullis, Appl. Phys. Lett. 61, 228 (1992).

${ }^{4}$ C. Jaekel, R. Barth, H. Roskos, and H. Kurz, J. Vac. Sci. Technol. A 12, 2830 (1994)

${ }^{5}$ P. Marcoux and P. Foo, Solid State Technol. 24, 115 (1981).

${ }^{6}$ T. Benson et al., J. Vac. Sci. Technol. B 14, 483 (1996).

${ }^{7}$ H. Maynard and N. Hershkowitz, J. Vac. Sci. Technol. B 13, 848 (1995).

${ }^{8}$ A. Stano, C. Coriasso, and G. Meneghini, Semicond. Sci. Technol. 11, 968 (1996).

${ }^{9}$ T. Vincent, P. Khargonekar, and F. J. Terry, J. Electrochem. Soc. 144, 2467 (1997).

${ }^{10}$ T. Vincent, P. Khargonekar, and F. J. Terry, IEEE Trans. Semicond. Manuf. 10, 42 (1997).

${ }^{11}$ P. Heimann and R. Schutz, J. Electrochem. Soc. 131, 881 (1984).

${ }^{12}$ E. Klein and W. Ramirez, AIChE J. (in press).

${ }^{13}$ E. Klein and W. Ramirez, J. Vac. Sci. Technol. A 18, 166 (2000).

${ }^{14}$ A. Golubev and A. Chekhovsky, Opt. Eng. (Bellingham) 36, 2229 (1997).

${ }^{15}$ Hewlett-Packard, Application Note No. 325-12, October 1997 (unpublished).

${ }^{16}$ N. Riza, Rev. Sci. Instrum. 67, 2466 (1996).

${ }^{17}$ Y. Zhao, T. Zhou, and D. Li, Opt. Eng. (Bellingham) 38, 246 (1999).

${ }^{18}$ C.-C. Huang, Opt. Eng. (Bellingham) 23, 365 (1984); see also C.-C. Huang, U.S. Patent No. 4,848,908 (1999).

${ }^{19}$ Handbook of Optics, edited by M. Bass (McGraw-Hill, New York, 1995).

${ }^{20}$ O. Heavens, Optical Properties of Thin Solid Films (Dover, New York, NY, 1965).

${ }^{21}$ H. Jennewin, H. Gottschling, T. Ganz, and T. Tschudi, Proc. SPIE 3677, 1009 (1999). 